\title{
Advance System for Heart Rate Monitoring Based on Internet of Things (IoT)
}

\author{
Arpita Suri \\ Department of Biochemistry Shree Guru Gobind Singh \\ Tricentary University, Gurugram, Haryana, India \\ Corresponding author email: arpita_fmhs@sgtuniversity.org
}

\section{ABSTRACT}

Heart rate monitoring plays an important role in maintaining health of heart. Humans of different ages have specific heart rate thresholds optimum and minimum values; the heart rate control system is sufficiently compatible to tackle this condition. In this article, an IoT-based device that can track the heartbeat from sensor output data \& a hardware system comprised of a pulse sensor is developed. In addition, a warning system is attached to provide notification when the pulse falls below or rises above a threshold or set stage. The doctor receives the warning message from a cell phone programmed. Using this programmed application, doctors will remotely monitor the patient's pulse data. The heart rate of the patient will be monitored by medical personnel such as nurses or doctors who are on duty at the hospital. The patient's pulse and other personal records are saved in the database and can be used for further monitoring of the patient's health status.

\section{KEY WORDS: HEARTBEAT, HEART RATE, INTERNET OF THINGS, SENSORS, MICROCONTROLLER.}

\section{INTRODUCTION}

Monitoring heart rhythm is important for patients, sports, and average people because it controls the heartbeat state, or heart rate. There are many methods for calculating pulse rate, the best of which is electrocardiography, but the simplest way to track heart rate is to use a Heartbeat Sensor. Heartbeat sensors are available in various ranges of shapes and sizes, and they regulate the heartbeat in real time. Sensors for recording heartbeats can be used in smart wrist bands, cellular phones, chest belts, and a variety of other devices. The heartbeat is measured in beats per minute, or bpm, which means how many times the heart contracts or expands in a minute.(M. Patil et. al. (2019).

In today's world, more than millions of people every year lose their lives due to serious heart related disease called

Biosc Biotech Res Comm P-ISSN: 0974-6455 E-ISSN: 2321-4007

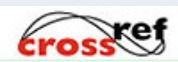

Identifiers and Pagination

Year: 2021 Vol: 14 No (6) Special Issue

Pages: 401-404
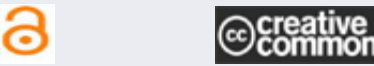

This is an open access article under Creative

Commons License Attribn 4.0 Intl (CC-BY). DOI: http://dx.doi.org/10.21786/bbrc/14.7.84

\section{Article Information}

Received: $15^{\text {th }}$ May 2021 Accepted after revision: $19^{\text {th }}$ July 2021 cardiovascular diseases. Generally, heart related disease patients will live at their home and take suggestions regarding service related to healthcare when patients feel sick. However, in most cases, people do not get ill unless they are at the end of the disease's progression, by which point the harm is permanent. Many people die before they can get better care. As a result of improved heart disease healthcare efficiency, the rate of mortality will be reduced and the optimistic mode of health care will become more common, which ensures that the real-time health status of patients can be tracked by specialist doctors, and physicians will advise whether to deliver healthcare services depending on the real-time status of patients. The real-time framework is an essential component of this advanced healthcare mode system(C. Li et. al. (2017).

Fig.1 shows the overall working of the proposed system from sink to web server and web server to physician. Human body most extensive organs are the heart. It generally works like a circulatory pump for transmission blood \& oxygen across the body. A heartbeat is described as a portion of the heart's pumping operation lasting around one second. Pumping is caused by the heart's relaxation. The Sino Atrial node sends an electrical signal when blood is found in the upper most heart chambers\& allowing the heart atria to contract. The 
blood is then forced into the mitral and tricuspid valves by contraction; this related pumping stage mechanism is known as diastole. When the ventricles of heart are fully occupied with blood, the next move begins.

Figure 1: Sequence systematic diagram of heart beatrecording system(D. S. Kumar et. al. (2020).

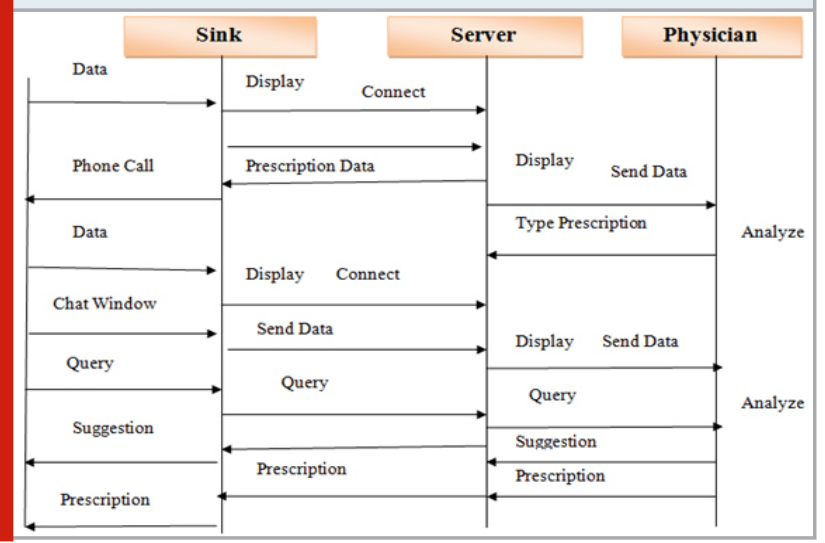

Electrical signals produced by Sino Atrial nodes reach the ventricle, causing it to contract. Health issues, such as heart disease, are still prevalent in today's world. Diseases of the heart are a leading cause of death of both men and women. Per year, nearly 1 million people die as a result of heart failure. Heart rhythm is a vital factor in the proper operation of the heart. As a result, controlling heart rate plays a vital role in observing heart performance and, as a result, improving heart health(T. V. Sethuraman et. al. (2019).

Using IoT, this research paper addresses the issue of heart rate control and identification of abnormalities in the heart system. The management of certain heart conditions today necessitates constant and long-term care. IoT aids in ongoing and long-term traditional surveillance schemes for an effective scheme, by supplying critical information about the patient's health that is remotely accessed by doctor at any moment and from any location through the internet. Furthermore, medical staffs such as nurses or doctors who is on duty at the moment will track the patient's heart rate using the actual time surveillance proposed system.

In addition, a deterrent system is linked to the heart rate control, which sends a Notification to the physician if the patient's pulse falls below or rises above a predetermined level. GPS technology is used in the proposed method to map the location of the system and the patients. The suggested system's database would also store information about the patient's heartbeat as well as other healthrelated evidence, and the doctor would be able to use all of this information to track the patient's heart condition. Recognizing the condition in the early phases is critical to protecting the patient from any problems in the future( $\mathrm{T}$. V. Sethuraman et. al. (2019)).

Literature Review: The Internet of Things (IoT) is becoming increasingly relevant in people's everyday lives. IoT-based system involving computing systems, automated and mechanical computers, objects, and individuals with special IDs, as well as knowledge transfer over a network without any physical interference with human-to-human or human-to-computer interaction. Physical structures that are no longer cut off from the physical world and can be managed from anywhere using Internet services. An Internet of Things (IoT) computer is an electronic device that interacts between a system and a Web server. The use of IoT allows data to be collected wirelessly from Web servers. The following essential metrics are relevant to the patient's heart condition measurements: heart rate, variability of heart rate, and body temperature. All of these heart-related parameters must be tested on a daily basis to ensure life protection(V. R. Parihar et. al. (2017).

The sensor is linked to a microcontroller, which allows for heart rate monitoring, measurements, and data transmission across the internet. The approved person has the ability to restrict the number of heart beats per minute. After setting these thresholds, the proposed device start observing the patient's heart beat \&whenever the patient's heart rate is more than the threshold fixed limited value, the proposed system transmits information to the microcontroller, which then communicates this information on the web and notifies the physicians(T. W. Nam et. al. (2007).

Automatic heart rate monitoring system based on a microcontroller Mamun AL, Ahmed N, ALQahtani Journal OF Theory and Applied Science, from fingertip Heart-rate signals were detected from ears or fingertips in this study using the IR TX-RX module, which amplified the observed data in order to translate all observed data to an accurate scale. A low pass filter is used to filter out inherent noise. A microcontroller measured and showed the detected signals on the LCD. The proposed heart health monitoring system is run by a microcontroller with a programmable algorithm. When the results of this process were compared to those of the manual examination involving heart rate counting, they were found to be satisfactory. The new scheme is appropriate for hospital, families, private treatment group, sports healthcare, and other medical applications. This article, however, does not propose a scheme for remotely tracking patients(Sahana S Khamitkar et. al. (2020).

This proposed research paper explains how to install recording of heartbeat and heart disease prevention systems using IoT. There is an uptick in the number of heart disease patients and heart attacks at the moment. The sensor is linked to a microcontroller, which allows for heart rate monitoring, measurements, and data transmission across the internet. The approved person has the ability to restrict the number of heart beats per minute. The proposed system transmits information to the microcontroller, which then communicates this information on the web and notifies the physicians(T. V. Sethuraman et. al. (2019).

The two most prevalent circulatory diseases are stroke and heart disease. A heart attack is a condition that happens when arteries get clogged as a result of blood supply damage to the heart or when blood flow is blocked, causing heart muscle damage or, in many cases, death of the heart muscle. A stroke, also known as a brain attack, is caused 
by a blockage or disruption of the brain's blood flow. Heart strokes are not called heart diseases in and of themselves. Blood clots form in the blood and migrate to the brain, obstructing the artery, resulting in a stroke(T. V. Sethuraman et. al. (2019).

The salient feature of this proposed research paper is:

1. The working of the proposed system is to real-time monitoring \& detection of the human heartbeat rate.

2. The proposed system displays real-time heartbeat rate of patients on LCD monitors.

3. The proposed system transfers the collected data of patients to the web data server for observation \& analysis.

4. Practitioners related to medical field or specified doctors can remotely diagnose \&monitor as well as control the patient health issue(S. Abba et. al. (2019)).

\section{METHODOLOGY}

The proposed heart rate monitoring system provides an efficient monitoring system which is designed to monitor the patient heart beat on real time $\&$ without the presence of any medical staff at any place with the help of IOT technologies. Inside the house patient behavior observation and analysis are involved in this proposed system, even the patients can be observed in any condition without any restrictions. Because it is easy to handle and cost efficient anyone can use this proposed system easily \& its operation is also user-friendly(M. Patil et. al. (2019).

Figure 2: Systematic chart of proposed system(Sahana $S$ Khamitkar et. al. (2020).

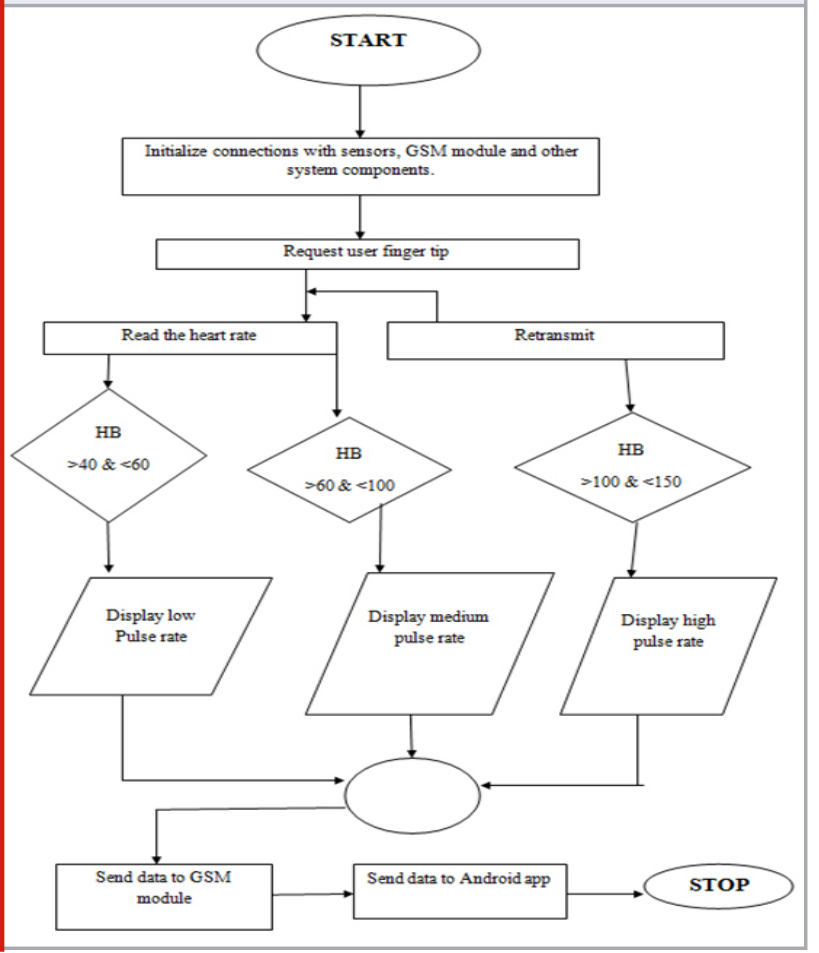

Fig. 2 shows the working sequence diagram of proposed system firstly initialized all the sensors and hardware system, the sensor reads the heartbeat information if heartbeat is less than $40 \mathrm{bpm}$ and higher than $60 \mathrm{bpm}$ display low pulse rate or send notification to user mobile same process repeat if pulse rate is higher than $150 \mathrm{bpm}$ and less than 100 bpm(Sahana S Khamitkar et. al. (2020).

Data Analysis: The relevant information about various factors which make different people's heartbeat rate at rest time is different. In normal circumstances, an adult's resting heartbeat rate should be 72 beats per minute. A normal child's heartbeat rate ranges from 70 to 120 beats/ min (bpm), whereas a normal adult's heartbeat rate ranges from 60 to 100 beats/min(bpm). Similarly, the heartbeat of older people ranges from 65 to 125 beats/min(bpm) depending on their age (patients aged 60 and up). Other significant factors that influence heart rate include body size, individual activity level, and many others.

Instrument: Heart rate sensor: Sensor related to heart rate measures pulse waves, that changes in the volume of a blood vessel which generated when blood is pumped by the heart. Waves related to pulse are sensed by measuring the change in volume of blood transmission using an optical sensor \& green LED.

Arduino Unit: The Arduino Unit is an open source electronic platform to read input light from the sensor and provides information to GSM for wireless communication.

GSM system: GSM is an international standard for wireless telephones. It is a communication system that stands for the Global system for wireless communications(B. Mallick et. al. (2016)).

\section{RESULTS AND DISCUSSION}

The proposed method herein presents the identified findings from the recommended sample system. The sensor that measures heart rate transmits heart related information \& the collected heart related data to the microcontroller. Further microcontroller decodes the gathered information \& further transmits it to the user's mobile device or display on screen for depiction. The collected data is further transmitted to the GSM module \& passed to the thing talk IoT network through a convenient internet link. The proposed heart rate monitoring system is run by a microcontroller with a programmable algorithm. When the results of this process were compared to those of the manual examination involving heart rate counting, they were found to be satisfactory. The new scheme is appropriate for hospital, families, private treatment group, sports healthcare, and other medical applications. After setting these thresholds, the proposed system begins to monitor the patient's heart rate. As the patient's heart rate exceeds the set limit, the proposed system sends an alert to the microcontroller, which then sends the alert to the doctors over the internet. Furthermore, the proposed method employs the same alerting procedure for lower heartbeats.Thus, Patients can track their heart rate as well as get real-time notifications of a heart attack from everywhere, allowing them to conserve time. To access heartbeat records, a patient must sign in to their think talk account \& display their observed data, further which is visualized in the form of a graph. 


\section{CONCLUSION \& IMPLIFICATION}

This research paper considers the prototype \& application of particular patient heart-beat rate controlling \& tracking device based on IoT technologies and a heart pulse sensor to measure heartbeat. The rate of heartbeats is monitored and recorded as data signals. The microcontroller then processes the detected data \&further transfers it to the communication module for uploading on the web server platform for further review and tracking. The collected information is further processed in real time \&save the collected information in the database. The primary aim of this research paper is to implement and develop a low-cost, user-friendly system for managing and tracking human heartbeat rates. This approach is used to construct an integrated sample that could be used to manage and track heart-related issues.

\section{REFERENCES}

Abba, S. and Garba, A.M., 2019. An IoT-Based Smart Framework for a Human Heartbeat Rate Monitoring and Control System. In Multidisciplinary Digital Publishing Institute Proceedings (Vol. 42, No. 1, p. 36).

Li, C., Hu, X. and Zhang, L., 2017. The IoT-based heart disease monitoring system for pervasive healthcare service. Procedia computer science, 112, pp.2328-2334.
Mallick, B. and Patro, A.K., 2016. Heart rate monitoring system using finger tip through arduino and processing software. International Journal of Science, Engineering and Technology Research (IJSETR), 5(1), pp.84-89.

Nam, T.W., Cho, J.M. and Noh, H.J., 2007. Design of a Wireless Heart Rate Monitoring System for Rehabilitation Patients. In 3rd Kuala Lumpur International Conference on Biomedical Engineering 2006 (pp. 625-627). Springer, Berlin, Heidelberg.

Parihar, V.R., Tonge, A.Y. and Ganorkar, P.D., 2017. Heartbeat and temperature monitoring system for remote patients using Arduino. International Journal of Advanced Engineering Research and Science, 4(5), p.237161.

Patil, M., Madankar, A. and Khandait, P.D., 2019, March. Heart rate monitoring system. In 2019 5th International Conference on Advanced Computing \& Communication Systems (ICACCS) (pp. 574-576). IEEE.

Patil, M., Madankar, A. and Khandait, P.D., 2019, March. Heart rate monitoring system. In 2019 5th International Conference on Advanced Computing \& Communication Systems (ICACCS) (pp. 574-576). IEEE.

Sethuraman, T.V., Rathore, K.S., Amritha, G. and Kanimozhi, G., 2019. IoT based system for heart rate monitoring and heart attack detection. Int. J. Eng. Adv. Technol.(IJEAT), 8(5), pp.1459-1464. 